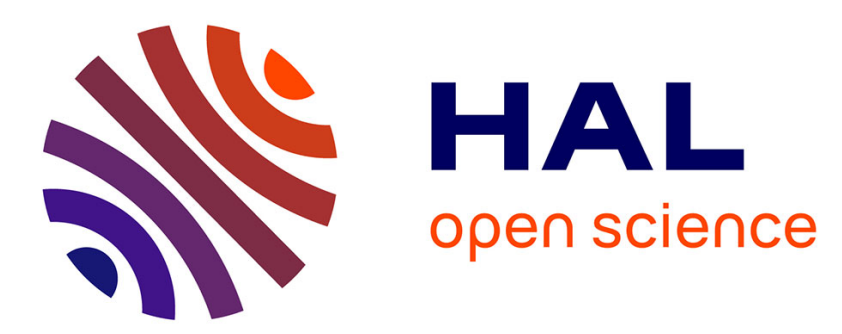

\title{
Former les professeurs des écoles à la réception des oeuvres chorégraphiques contemporaines : analyse épistémique et obstacles à l'expérience
}

\author{
Alexandra Arnaud-Bestieu
}

\section{- To cite this version:}

Alexandra Arnaud-Bestieu. Former les professeurs des écoles à la réception des oeuvres chorégraphiques contemporaines : analyse épistémique et obstacles à l'expérience. Recherches \& éducations, 2019. hal-02461369

\author{
HAL Id: hal-02461369 \\ https://hal.science/hal-02461369
}

Submitted on 30 Jan 2020

HAL is a multi-disciplinary open access archive for the deposit and dissemination of scientific research documents, whether they are published or not. The documents may come from teaching and research institutions in France or abroad, or from public or private research centers.
L'archive ouverte pluridisciplinaire HAL, est destinée au dépôt et à la diffusion de documents scientifiques de niveau recherche, publiés ou non, émanant des établissements d'enseignement et de recherche français ou étrangers, des laboratoires publics ou privés. 


\title{
Former les professeurs des écoles à la réception des œuvres chorégraphiques contemporaines : analyse épistémique et obstacles à l'expérience
}

\begin{abstract}
Alexandra Arnaud-Bestieu
Aix-Marseille Université - ADEF (EA 4671)

Les instructions officielles de 2015 pour l'école élémentaire accroissent encore un peu plus la mission du professeur des écoles (PE) comme passeur culturel, demandant aux PE de nouveaux savoirs et de nouvelles compétences didactiques et pédagogiques. Cette polyvalence toujours plus élargie n'est pas sans mettre l'enseignant en difficulté, en particulier pour des disciplines comme la danse. Nous nous interrogerons dans cet article la réception des æuvres chorégraphiques en tant que praxéologie ainsi que ses tenants expérientiels. Pour contribuer à la compréhension et à l'innovation en termes de formation chorégraphique de l'enseignant polyvalent, nous proposerons l'analyse d'une recherche-intervention auprès de 132 étudiants Master 1 MEEF (PE) en nous focalisant sur les indices nous permettant d'approcher les différentes expériences qu'ils ont pu traverser et les obstacles épistémiques à prendre en compte : Quels sont les caractéristiques principales de leur réception avant tout travail chorégraphique? Quels indices d'expérience au moment de la pratique? Quels effets du temps de pratique sur la réception? Avec quelles limites? MOTS-CLÉS : formation, réception, œuvres chorégraphiques, danse, professeurs des écoles, expérience

The official French instructions (2015) for the primary school increase the mission of the teacher as cultural mediator. This mission require new knowledge and new didactic and pedagogic skills. This is a difficulty,particularly for secondary fields, like dance.In this paper, we question the reception of three works (Jiri Kilian,Pina Bausch, A-T de Keersmaker) and the dimensions of the experience. It seeks a better comprehension and innovation for a cultural and choreografic knowledge in teacher training. This paper presents a research based on an intervention.
\end{abstract}

Keywords : teacher training, dance, choreografic work, reception, experience 
Les instructions officielles de 2015 pour l'école élémentaire française accroissent encore un peu plus la mission du professeur des écoles (PE) comme «passeur culturel» devant faire accéder les élèves au patrimoine de l'humanité, "transmettre une culture existante sans cesser de l'interroger, sans cesser de l'interpeller, sans cesser de trouver des moyens de la rendre accessible, humaine, partageable » (Zakhartchouk, 2005 ; p 3). Cette mission demande aux PE de nouveaux savoirs et de nouvelles compétences didactiques et pédagogiques et cette polyvalence toujours plus élargie n'est pas sans mettre l'enseignant en difficulté, en particulier pour des disciplines comme la danse (ArnaudBestieu, 2016) : sa quasi absence dans les cursus de scolarité et la très faible visibilité sociale des œuvres chorégraphiques (Germain-Thomas et Pagès, 2014) - ainsi que sa polylocalisation dans les programmes sans jamais constituer une discipline à part entière - accroît la médiocrité des rapports qu'entretiennent les enseignants à l'univers chorégraphique. Les enseignants sont donc amenés à devoir « passer» une culture chorégraphique qu'ils n'ont pas ou peu, ou à y renoncer.

Nous interrogerons dans cet article la réception des œuvres chorégraphiques ; comme une praxéologie (Chevallard, 1999) ainsi que les tenants expérientiels de celle-ci. Ainsi, nous poserons l'hypothèse que recevoir une œuvre chorégraphique constitue une potentielle expérience esthétique (Dewey,1934 ed. 2005) s'appuyant sur les expériences perceptives passées mais aussi sur l'expérience corporelle (Merleau-Ponty, 1945; Huet et Gal-Petitfaux, 2011) et sensible de son propre corps dansant. Pour contribuer à la compréhension et à l'innovation en terme de formation chorégraphique de l'enseignant polyvalent, nous proposerons l'analyse d'une recherche-intervention auprès de 132 étudiants Master 1 Métiers de l'Enseignement, de l'Education et de la Formation (MEEF) en nous focalisant sur les indices nous permettant d'approcher les différentes expériences qu'ils ont pu traverser et les obstacles épistémiques : Quels sont les caractéristiques principales de leur réception avant tout travail chorégraphique? Quels indices d'expérience au moment de la pratique ? Quels effets sur la réception? Avec quelles limites ? Répondre à ces questions nous permettra de mettre en lumière les obstacles épistémiques relatifs au « savoir recevoir une chorégraphie » et de proposer des pistes pour la formation.

\section{Réception et expérience}

Selon la littérature, la réception d'une œuvre chorégraphique pose différents problèmes. Tout d'abord, la faible diffusion des œuvres chorégraphiques dans la société et le corps mis en avant quand c'est le cas - corps performant, idéal - ne permettent pas une acculturation par les médias (GermainThomas et Pagès, 2014). Lorsque le néophyte ${ }^{1}$ est ainsi confronté à des œuvres contemporaines, il se retrouve face au syncrétisme et à l'instantanéité de formes corporelles, scénographiques et dramaturgiques (Guisgand, 2006 ; Bernard, 2001) que rien ne l'a préparé à démêler. La relation à l'œuvre de chacun est alors fonction « de la culture auquel le sujet appartient, de son propre cadre de référence, de sa "mémoire esthétique", de sa perception singulière du monde - notamment au niveau de sa sensibilité kinesthésique - et enfin de son histoire personnelle et collective » (Montaignac, 2014, p.2). Face à l'œuvre, le spectateur reçoit la danse à travers le prisme de ses références culturelles (au sens du cognitif) mais aussi de façon charnelle au sens phénoménologique du terme. Or, la (ou les) danse(s) dite(s) «contemporaine(s) $»^{2}$ met(tent) en scène des corps s'éloignant de l'image de ce que doit être un corps dans la société. Laban (1947 ed. 2003 ; 1950 ed. 1994), tout comme Mauss (1936) avant lui, ont défini la pression quasi-coercitive qui pèse sur les corps dans toute société ou groupe humain. L'uniformité du comportement corporel est vécue comme indispensable à la stabilité de la communauté. Il s'agit alors pour l'artiste de prendre son indépendance vis-à-vis des gestes reconnus par celle-ci, ceux-là même qui participent à définir une notion commune de ce qui est beau. Ainsi la danse contemporaine peut susciter incompréhension et rejets qui ne dépassent pas la critique d'homologation au sens de Mayen (2005) : il s'agit alors de « vérifier l'appartenance des ouvres à un

1 Nous utilisons le terme « néophyte » pour indiquer l'absence de réelle praxéologie, par opposition à « expert» (comme il est d'usage pour distinguer l'expertise des enseignants (Altet, Baillet, Carnus, etc)). C'est aussi le terme utilisé par Dewey. Dans cet article, il s'agit de notifier par ce terme le caractère très sommaire du rapport aux savoirs chorégraphiques des étudiants considérés.

2 Nous utilisons le pluriel ici pour porter deux idées : la première est de rendre compte de la pluralité des corps et des démarches au sein de ce que l'on regroupe comme étant de la danse contemporaine ; la deuxième est que, comme l'ont montré différent travaux (Arnaud et Arnaud-Bestieu, 2013; Fratagnoli, 2010) la problématique des corps dans les démarches dites contemporaines se pose aussi pour les danses dites « traditionnelles » (flamenco, danse indienne). 
courant repéré ; éventuellement les valider et sanctionner selon pareil critère ». (Mayen, 2005, p.46). Entre critique d'homologation et critique poreuse des œuvres (Mayen, 2005), la réception du spectacle chorégraphique est fonction du cadre de référence du spectateur, cadre émergeant de son histoire singulière, de sa mémoire esthétique et de sa sensibilité kinesthésique (Montaignac, 2014). Pour un danseur, "il paraît naturel de se frayer un chemin à travers cet écheveau de formes, de signes et de dispositifs en prenant le mouvement dansé comme fil conducteur et comme point de focalisation du regard» (Guisgand, 2006, p.118). Au-delà et en sus des aspects intellectuels et discursifs autour de l'œuvre, le corps y est central, corps sensible à la fois outil et support d'expression, d'émotions qui se font contagieuses, de corps à corps. L'œuvre chorégraphique contemporaine se construit sur ces deux dimensions : intellectuelle (concept, narrativité, références, etc.) et purement charnelle (au sens phénoménologique). La danse contemporaine s'éloignant de la narrativité et mettant en scène des émotions et concepts en utilisant le corps comme outil et comme finalité, un accueil poreux (Mayen, 2005) consiste en une expérience alliant agir et éprouver (Dewey, 1934 ed. 2005) qui sollicite avant tout la sensation et la mémoire kinesthésique (Montaignac, 2014 ; Guisgand, 2006). Comme l'écrit Dewey, la réception n'est pas un acte passif et sollicite des "canaux de réponses indirectes et collatéraux » issus d'une éducation esthétique reposant en partie sur une "préparation motrice » (Dewey, 2005, p.176) :

«Savoir ce qu'il faut regarder et comment le voir demande que l'équipement moteur soit prêt à cela. (...) Celui qui connaît la nature du rapport qu'il y a entre le jeu des mains du pianiste et la production de la musique qui en est issue entendra ce que le néophyte n'entend pas. » (ibid, p. 176)

Il s'agit bien pour Dewey de mettre en avant le lien entre l'expérience esthétique lors de la réception d'une œuvre d'une part et, d'autre part, les expériences esthétiques lors d'engagements dans la pratique et lors de réelles expériences de réception passées, où le sujet s'est vu transformé par des éprouvés intenses. Or, une expérience réelle est définie comme un moment de conscience et se démarque des expériences qui n'en sont pas en ce qu'elle engage tout l'être qui sort de la stase et entre en maturation.

Tout comme d'autres chercheurs travaillant sur la danse (Guisgand, $2006 ; 2014$ ) nous nous inscrivons donc dans une approche mettant conjointement en avant la notion pragmatique d'expérience et celle, phénoménologique (et plus récemment neuroscientifique) de perception. Cet ancrage épistémologique est à la base de nos choix didactiques de formation. En particulier, nous ne considérerons pas la réception de l'œuvre comme une exégèse reposant sur les seules connaissances et recherchant le sens de l'œuvre mais bien comme expérience sensible faisant appel à la mémoire émotionnelle du sujet et à son empathie kinesthésique, résonance motrice et incontrôlable entre la perception d'un geste et son revécu instantané. Il s'agit donc de faire pour comprendre (Guisgand, 2014), proposer une expérience (Dewey, 1934/2005), développant le sens kinesthésique des étudiants et donnant aux «impressions de l'ici et maintenant la consistance qui les transformera en connaissances » (Guisgand, 2006, p.14).

Cet article propose une mise en confrontation de ces postulats esthétiques et philosophiques avec une population définie et dans le cadre concret d'une recherche-intervention pour une compréhension fine et située du «savoir recevoir » la danse dont nous précisons qu'il n'est pas un savoir au sens de la bonne réponse mais bien une « puissance d'agir » (Sensevy, 2008).

\section{Construction du projet d'intervention et méthodologie de recherche}

Nous considérons que former (ici les futurs enseignants) consiste à nourrir et transformer leurs praxéologies - déterminant leur épistémologie pratique (Sensevy et Mercier, 2007) en situation d'enseignement - en faisant évoluer leur rapport aux savoirs (ici de la danse) et en créant du lien entre les blocs technique, technologique et théorique relatifs aux savoirs en question (Chevallard, 1999) : ainsi, à la suite de cet auteur, nous considérons que " recevoir un spectacle chorégraphique » peut être considéré comme un type de tâches dans une institution (par exemple le cours à l'ESPE) auquel correspond des techniques actualisant des savoirs (par exemple, prêter attention à l'écriture spatiale, au flux du mouvement, etc) et s'ancrant dans un discours explicatif technologico-théorique éclairant et justifiant ces techniques ( pour notre exemple, le fait qu'en danse contemporaine c'est la relation du corps aux paramètres fondamentaux du mouvement qui est au centre et non une esthétique préétablie). La réception est donc considérée dans cet article comme potentielle expérience d'un sujet donné à un moment donné mais aussi comme agrégat de savoirs implicites. Ce lien entre Dewey et 
didactique nous parait d'autant plus pertinent que l'auteur s'est intéressé à l'expérience artistique (1934) mais aussi à ses conditions d'émergence (1938), ce que nous pourrions appeler aujourd'hui dimensions didactiques d'aménagement du milieu.

Alors que peu de travaux sont consacrés à la formation des enseignants aux savoirs de la réception chorégraphique, notre travail fait suite à la thèse de Montaud (2014) qui montre (entre autres choses) l'importance de l'épistémologie pratique du professeur pour que les situations d'apprentissage mènent les élèves à une posture de spectateur. Alors que ce travail se focalise sur un seul enseignant sur des temps longs ponctués de temps de formation, nous nous intéressons à une recherche-intervention courte avec de nombreux participants et tentons d'approfondir l'analyse de l'impact de la pratique sur les savoirs de la réception (Guisgand, 2007 ; Montaud, 2014).

Notre propos n'est donc ni de prétendre à une vérité sur les œuvres convoquées, ni de prôner un modèle de formation mais bien de faire un état des lieux du rapport aux savoirs avant toute pratique puis d'identifier les incidences et les limites d'une initiation à la danse contemporaine et, ce faisant, de proposer une analyse épistémique de la réception : Pourquoi la réception ne va-t-elle pas de soi ? Quels en sont les obstacles inhérents ${ }^{3}$ et les savoirs à construire ?

Nous avons opté pour une recherche exploratoire à partir de l'hypothèse d'un certain impact de l'expérience de la danse par la pratique sur la réception d'œuvres. Son ambition est, en interrogeant leurs ressentis ainsi que leur réception d'extraits d'œuvres avant et après les ateliers de pratique chorégraphique, d'apporter des éléments de réponse aux questions suivantes : $\mathrm{Y}$ a-t-il une évolution du regard ? Sur quel(s) aspects(s) ? Avec quelle(s) limite(s) ? Quelle(s) piste(s) pour la réflexion relative à la formation? Le repérage fin des évolutions et des limites nous permettra non seulement de mieux comprendre les savoirs inhérents à la réception mais aussi d'en clarifier les dimensions perceptives, culturelles, émotionnelles pour réfléchir à la formation. Il s'agit donc d'une approche didactique visant à mettre en lumière l'émergence de l'expérience (au sens de Dewey) et l'évolution des praxéologies.

\subsection{Terrain et contraintes}

Cette recherche-intervention a été proposée à 132 étudiants en Master MEEF (Métiers de l'Enseignement, de l'Éducation et de la Formation) dans le parcours dédié au Professorat des Écoles, 121 femmes et 11 hommes, issus de licences variées (37\% Lettres et Langues, 31\% Sciences de l'Education,9\% Eco-Gestion, 9\% STAPS, 5\% Sciences de l'Homme, 5\% Sciences et technologies, 4\% sans licence). Parmi eux, 1 déclare avoir reçu un court enseignement en danse avant le bac, 8 durant leur licence STAPS (15 heures) en danse hip-hop et seuls 2 parmi les 132 ont une pratique amatrice (1 en contemporain, 1 en modern' jazz). Ces données nous autorisent à considérer cette population comme néophyte. Ce recueil de données par entretiens de groupe a permis de lier une approche qualitative (centrée sur le discours in situ) et un souci de représentativité des participants en termes de nombre et de diversité. Cependant, une recherche dans un tel contexte n'est pas sans impliquer des contraintes puisqu'il s'insère dans un projet d'enseignement : nous ne pouvions pas proposer plus de 4 heures de pratique et le choix des situations et de leur agencement avaient aussi pour objectifs de permettre aux étudiants de comprendre les éléments majeurs de la danse contemporaine attendus pour le concours ${ }^{4}$ et de s'approprier par la pratique des situations pouvant être proposées à l'école.

\subsection{Choix des situations de pratique}

Ayant comme objectif de formation de faire vivre aux étudiants une expérience où l'agir et le percevoir permettent un travail de conscience (Dewey, 1934/2005) pour ensuite analyser du point de vue de la recherche ce qu'une telle expérience peut amener en termes de savoirs relatifs à la réception, nous avons fait le choix d'une immersion dans les situations comme pratiquant. Très exigeante quant à la recherche par les étudiants d'un mouvement le plus juste possible en lien avec les consignes $^{5}$ (cf.

3 Nous renvoyons ici le lecteur à la notion d'obstacle définie comme structures et modes de pensée qui font résistance dans l'apprentissage (Reuter et al., 2007) et plus particulièrement comme obstacle épistémique, c'est-à-dire lié à la structure et à l'histoire des savoirs eux-mêmes (Brousseau, 2003).

4 Les sujets sont relatifs à la construction par les élèves du rapport au temps, à l'espace et à la relation en particulier dans le mouvement dansé. Il s'agit aussi de bien faire la différence entre les différents types de tâches que sont l'improvisation, la composition et le travail des formes.

5 Dans le même temps, cette exigence est aussi ce que je souhaite transmettre au futurs enseignants, convaincue, suite à Laban (1947 ed. 2003) que c'est cette recherche qui fait évoluer conjointement le rapport sensible à la motricité et 
Annexe 1), nous avons proposé une alternance entre situations collectives, d'improvisation ou de composition, et situations lors desquelles les étudiants ont été amenés à alterner les rôles de danseur et de spectateur (de leur pairs). Le choix des situations n'est pas en lien direct avec les extraits (mouvements, styles ou procédés chorégraphiques) ; il s'est agi de travailler sur le sensible (Garcia, Cogerino, Fouilhoux, 2013) entendu à la fois comme travail sur la kinesthésie, sur l'expérience du corps sensible (en particulier dans le travail sur le ralenti), mais aussi sur le vécu de l'expérience et le focus attentionnel (lors des échanges). Les consignes étaient alors de s'exprimer d'abord sur son ressenti en tant que danseur en termes de difficultés ressenties et d'aisance, de préférence dans le ressenti (entre mouvement ralenti et accéléré par exemple) et de l'état émotionnel qui a pu être expérimenté. Ensuite, il s'agissait de s'interroger et d'échanger sur la façon de regarder et de recevoir la danse des autres (attirance du regard, préférences quant aux différentes qualités de mouvement, état émotionnel). Ces moments d'échanges ont été enregistrés en audio.

\subsection{Choix des extraits vidéo}

La recherche ayant comme objet de décrire et comprendre 1 'impact d'une potentielle expérience à partir de ces situations vécues sur la réception d'œuvres chorégraphiques, il nous a fallu choisir les œuvres en question. Tout d'abord, notons que nous sommes conscients de la différence de qualité entre extraits vidéo et pièce chorégraphique in situ; cependant, le terrain nous a seulement permis la projection sur grand écran en salle de cours. De plus, travailler sur les pièces chorégraphiques aurait eu du sens pour ne pas morceler le propos artistique et pour prendre l'œuvre dans son entier, sa cohérence. Cependant, les contraintes temporelles nous ont mené à la sélection d'extraits ${ }^{6}$, ce qui n'a pas été sans poser question: Quel chorégraphe? Quelle époque ? Quel spectacle ? Quel passage ? Ainsi, nous avons tenté de choisir trois extraits dont l'accès pour des néophytes posent des problèmes distincts en termes de distance au corps mis en valeur dans notre société - bienséant et performant (Mauss, 1935 ; Germain-Thomas et Pagès, 2014) - d'émotions véhiculées et de radicalité de l'écriture. Ainsi, voici les trois extraits choisis et les motifs de leur sélection :

- V1 : Jiri Kylian, Six dances (in Black and white ballets, Hans Hulscher, 1997),

Cet extrait a été choisi car l'écriture de Kylian mêle un usage du corps dit «contemporain » (danse non codifiée, mobilité de la colonne vertébrale, rapport à la gravité et au sol) et « néo-classique » (figures codifiées, prégnance de certaines formes esthétisantes) tout en jouant sur des costumes théâtraux, une musique classique (Mozart) et une dimension burlesque très prononcée. Ces caractéristiques nous ont amené à considérer cet extrait comme accessible par la corporéité mise en avant et les émotions véhiculées (humour) et à le proposer en premier.

- V2 : Pina Bausch, Café Müller (1985), trio en silence et début de Didon et Enée ${ }^{8}$

Repère dans l'histoire de la danse, nous avons choisi cet extrait car il mêle radicalité des corps mis en avant - dans le sens où les corps n'y sont pas idéaux et performants - et dimension tragique où la prégnance d'émotions intenses est accentuée par l'univers sonore (silence et Purcell). Il nous a donc semblé que cette pièce pouvait à la fois être considérée pour des néophytes comme difficile à recevoir ou au contraire facile en fonction du cadre de référence de chaque étudiant.

- V3 : Teresa de Keersmaker, Rosas danst Rosas,(Thierry de Mey, 1997), scène des chaises ${ }^{9}$

Cet extrait se distingue des deux premiers : radical par l'usage du corps dansant (assis, prégnance du travail autour du buste, jeux de flux) et des processus chorégraphiques d'écriture (répétition, partition chorégraphique complexe) mais mettant en scène des corps rapides, performants bien que s'éloignant des conventions (lien à la sexualité). Ainsi, cet extrait peut paraître difficile d'accès en raison de son écriture et de sa distance aux formes esthétisantes mais met en scène, dans le même temps, des corps

l'élargissement de la palette des qualités de mouvement.

6 A posteriori, nous pensons que la proposition de pièces chorégraphiques entières n'aurait rien apporté, une majorité d'étudiants ayant déjà eu du mal lors du premier visionnage à rester attentive le temps des extraits...

$7 \quad$ Extrait du début à 4’56

8 Extrait $10{ }^{\prime} 55-22^{\prime} 50$

$9 \quad$ Extrait $12{ }^{\prime} 20-22^{\prime} 12$ 
volontaires, performants, extraordinaires généralement valorisés aux yeux des néophytes (GermainThomas et Pagès, 2014 ; Massot-Leprince, 2016).

Les trois extraits ont été vus à la suite les uns des autres avant le temps d'échange, partant du principe que l'absence d'une vidéo lors des échanges était une donnée en soi, tout comme l'insistance des étudiants sur un extrait. Rappelons que les trois extraits sont vus deux fois, la première avant tout enseignement, la seconde après les quatre heures de pratique.

\subsection{Recueil et analyse des données}

Afin d'approcher les composantes et obstacles épistémiques relatifs à l'expérience esthétique lors de la réception (résistances, évolution), nous avons fait le choix d'enregistrer en audio tous les temps d'échanges autour des ressentis, lors de la pratique et lors des visionnages, au sein du groupe de formation (environ 25 étudiants). Clairement informés qu'ils participaient là à une large recherche, chaque étudiant a d'abord écrit cinq mots pour chaque vidéo et les a ordonnés du plus important au moins important pour rendre compte de la réception de l'œuvre proposée. Cet écrit a pour fonction de préparer l'entretien et d'impliquer chaque individu dans un groupe élargi(Guillemette et Lapointe,2011), L'entretien de groupe (Baribeau, 2009), lors de laquelle la parole est librement distribuée par les étudiants eux-mêmes, nous paraît être intéressante tant du point de vue de l'intervention que de la recherche en permettant à la fois l'émergence d'une réflexion dépassant la seule subjectivité et menant à des «élaborations collectives $d u$ discours» (Guillemette et Lapointe,2011., p. 8) ; ainsi les propos qui s'affinent en se confrontant à ceux des autres, la pensée réfléchie trouvant ses fondements dans ces moments d'intersubjectivité que constitue le partage social, ici en particulier autour d'émotions (Terrien,2006)

La chercheure, abandonnant sciemment et ouvertement la posture de formatrice pour le recueil de données ${ }^{10}, \mathrm{~s}^{\prime}$ est limitée à trois types d'interventions :

- Pour les entretiens suivant les deux visionnages, le lancement s'est fait par la consignes « Exprimezvous librement sur les vidéos que vous venez de voir. ». Cette question très ouverte a été choisie sciemment pour ne pas orienter vers l'appréciation ou l'évaluation esthétique (Château, 2010) ${ }^{11}$.

- Pour les situations vécues. "Pouvez-vous me dire ce que vous avez ressenti en tant que danseur/en tant que spectateur? Qu'avez-vous apprécié ou au contraire pas apprécié, dans quel état cela vous at-il mis?»

- Lorsqu'aucun étudiant ne rebondit; la relance est alors une répétition de la consigne initiale ou la reprise de ce qui vient d'être dit par un étudiant.

\begin{tabular}{|l|l|l|}
\hline \multirow{2}{*}{ Décembre } & Premier visionnage & Visionnage des trois extraits \\
\cline { 3 - 3 } & & Ecrit (mots) \\
\cline { 3 - 3 } & Entretien de groupe \\
\hline \multirow{2}{*}{ Janvier } & Pratique & $\begin{array}{l}\text { Situations danseurs/spectateurs } \\
\text { Entretien de groupe }\end{array}$ \\
\hline \multirow{2}{*}{ Février } & Deuxième visionnage & Visionnage des trois extraits \\
\cline { 3 - 3 } & & Ecrit (mots) \\
\cline { 3 - 3 } & & Entretien de groupe \\
\hline
\end{tabular}

Protocole global

Après transcription, les verbatims d'entretien ont été condensés dans des tableaux (cf. Annexes 2 et 3 ), afin de repérer la récurrence de certaines thématiques alors que les transcriptions intégrales nous permettent d'analyser plus finement certains propos choisis. Nous nous centrons en effet sur une analyse de contenu des différents temps d'échanges en essayant d'y repérer les savoirs caractérisant le

10 Il a bien été précisé aux étudiants que ce temps n'était nullement évaluatif mais pourrait permettre de mieux réfléchir à la formation par l'analyse des échanges.

11 Château (2010) distingue l'appréciation esthétique, relation sensible à l'œuvre, de l'évaluation esthétique qui tente d'élucider les propriétés de l'œuvre. 
«cadre de référence» (Montaignac, 2014) des étudiants, en particulier dans sa fonction d'homologation, les ressentis, et les traces d'expérience (Dewey, 1934 ed.2005).

\section{La réception, entre obstacles et expériences}

\subsection{Premier visionnage : un état des lieux de la réception des ouvres chorégraphiques}

Les entretiens suivant le premier visionnage sont marqués, dans tous les groupes, par la prégnance de la critique d'homologation. Ainsi, la question centrale qui animent les échanges entre étudiants est la suivante : les vidéos 2 et 3 sont-elles des vidéos de danse? Et en cascade, qu'est-ce que la danse? Qu'est que je désire voir quand je suis amené à voir de la danse? Dans le groupe 2, ce débat - autour de ce qu'est la danse et de ce "qu'elle devrait être » selon certains étudiants - débute sans lien exprimé à l'une ou l'autre des vidéos pour ensuite s'attarder sur l'extrait de Café Müller (V2). Pour les groupes 1 et 4 cet entretien s'est articulé exclusivement autour de ce dernier alors que dans les groupes 3 et 5 l'extrait de Rosas danst Rosas (V3) a aussi été mis en question.

Dans le tableau (Annexe 2) nous avons condensé les thématiques abordées par les étudiants dans chaque groupe dans l'ordre chronologique pour observer comment les entretiens se sont construits. Alors que dans le G1, le discours d'homologation s'accompagne du partage de ressentis marqués par l'angoisse (ou l'ennui) face à l'extrait de Café Müller, celui des groupes 3, 4 et 5 se voit ponctués par des remarques relevant d'une réception plus poreuse (corps qui est musique (V2), émotion avant sens, métaphore de l'humanité/de la société, force du silence (V2), liberté d'interprétation, tristesse (V2), lien à la sexualité (V3)). Ces propos d'étudiants ponctuent alors les discussions sans les dominer, contrairement au G2 où les propos d'étudiants plus avertis face à la contemporanéité en arts dominent et qui, suite à la première intervention (d'homologation négative) tentent de définir les démarches artistiques en art contemporain. Ainsi, il faut attendre 17 minutes pour qu'une autre étudiante de ce groupe livre une réflexion relevant de l'homologation. Il nous semble donc que la dynamique au sein du groupe est fondamentale pour comprendre ces entretiens. En effet, le G1 ne voit aucun étudiant dont les propos s'éloignent de l'homologation ou d'une angoisse subie et c'est dans ce groupe que l'entretien est le plus court lors des deux visionnages (13'24' et 12' contre 22' en moyenne). Dans le même temps, nous pouvons aussi penser que le G2 est moins marqué par le discours d'homologation car les étudiants se situant à ce niveau de réception lors du premier visionnage ont certainement eu plus de mal à prendre la parole que dans les autres groupes, compte-tenu des propos plus avertis de quelques-uns de leurs collègues qui mobilisent ici une culture artistique générale, non spécifique à la danse - et lorsqu'ils le font, c'est avec une agressivité particulière. Leurs propos sont alors marqués par le sentiment d'agression et l'agressivité face aux extraits de Café Müller et Rosas danst rosas (" c'est n’importe quoi » $(\mathrm{G} 2)$, " comique de répétition, ridicule 》 $(\mathrm{G} 2)$, «arnaque » $(\mathrm{G} 2)$, "pas de sens » (G3), « mouvements mis bout à bout, ridicule » (G5)). D'autres expriment de l'ennui face à ce qui est pour eux incompréhensible (préférence pour danses "plus époustouflantes » (G3), chute dans le duo de Café Müller «drôle parce qu'elle tombe » puis lassitude (G4)). Chez d'autres certains étudiants, nous repérons une conscience de leur distance à l'art : «Loin de nous, difficile» (G2), «Inaccessible quand on n'a pas les codes»(G2), "Hermétique, il faut être initié»(G5), "Ouverture d'esprit nécessaire » (G5). Ces étudiants qui n'apprécient pas d'emblée les extraits se posent cependant la question de leur posture de récepteur. Enfin, rappelons que certains étudiants ont des propos soit relatifs à l'art en général (liberté du créateur et du spectateur en termes d'interprétation, subjectivité du spectateur, recherche de l'intention (G2), émotion avant sens (G3), interprétation personnelle (G4 et G5)), soit relatifs à leur ressenti (V1 : parodie, codes classiques cassés ; V2 : souffrance, métaphore société, inéluctabilité, tragique, force du silence, marionnettes, mise en avant de l'inconscient, rapport à l'autorité ; V3 : angoisse due à l'accélération, sexualité, hypnotique, métaphore humanité (décalage), folie, aliénation, camisole, violence, féminité stéréotypée) qui dénotent d'une réception plus poreuse, sensible au propos dramatique de l'extrait.

\subsection{Propos des étudiants lors de la pratique chorégraphique}

Les étudiants ont été amenés le mois suivant à vivre deux ateliers de 2 heures chacun autour de situations d'improvisation et de composition (Annexe 1). Nous voudrions observer brièvement la dynamique des échanges lors des moments d'alternance des rôles (danseur/spectateur de ses pairs) de façon à pouvoir les confronter aux propos lors du second visionnage. Nous nous centrerons sur les 
ressentis relevant de l'état émotionnel et de la réception. Dans tous les groupes, au moins un étudiant a ouvert la discussion sur un état agréable ou au contraire désagréable expérimenté en tant que danseur, en particulier en ce qui concerne le mouvement au ralenti : «c'est très agréable, on oublie tout » (G3, G5), « c'est vraiment planant» (G5), " $j$ 'ai adoré cet arrêt du temps » (G2), «ça aurait pu durer des heures » $(\mathrm{G} 4, \mathrm{G} 3)$,; «il fallait que ça se termine » (G2), "ça m'angoisse, je suis trop nerveuse pour $c ̧ a ! »(\mathrm{G} 1)$. Ces moments d'échanges nous ont permis d'aborder la subjectivité des ressentis face à ce rapport au temps extraordinaire En tant que spectateur, chaque groupe a accueilli plusieurs remarques sur la dimension poétique et sensible de ce qui était alors partagé : "on ne voit plus nos copains de classe, on voit des êtres sensibles» (G5), «c'est magique, on dirait que le temps s'arrête » (G2), «à la fois mon regard était attiré par ceux qui étaient au ralenti, et en même temps j'avais la sensation que je ne devrais pas regarder... c'est tellement... intime, ouais, intime » (G3). Soulignons que ces propos sont autant énoncés par des étudiants repérés lors du premier visionnage comme ayant une critique plus poreuse que par des étudiants n'ayant pas adhéré aux vidéos. Bien que nous restions très rapides sur cette partie de notre travail de recherche, ces propos tout comme notre propre ressenti lors de ces ateliers, nous font penser qu'une majorité d'étudiants a vécu une expérience à la fois corporelle, sensible et esthétique, inédite pour certains. Quel(s) effet(s) de cette expérience sur la réception d'œuvres chorégraphiques?

\subsection{Second visionnage : évolutions de la réception et limites}

Lors du second visionnage des extraits, un mois après les ateliers, la première prise de parole, dans chacun des groupes, a pour thématique le changement de regard entre les deux visionnages. Le biais possible serait que ces déclarations soient liées à une lecture par les étudiants d'une attente de notre part ; cependant, par la suite, certains propos vont dans le même sens alors que d'autres n'hésitent pas à revenir aux questions d'homologation, sans censure. Nous relevons (Annexe 3) deux types d'évolution constatée, celle des émotions et celle de la technique (regard et mots). Alors que l'entretien dans le G1 reste bref et doit être relancé pour atteindre 12 minutes, le G2 voit les échanges marqués par l'impact de la pratique pour comprendre la danse et en parler mais aussi par un débat d'homologation particulièrement intéressant en termes d'analyse épistémique de la réception. Les G3, G4 et G5 voient leur attention focalisée sur le rapport entre regard analytique et émotions. Pour faciliter la lecture de ces résultats nous les exposerons en deux parties : les changements relevant d'une réception plus poreuse et réflexive et les limites dans cette évolution.

\subsubsection{Incidence de la pratique sur la réception : vers une critique plus poreuse et réflexive}

Comparativement au premier visionnage, les étudiants s'éloignent de la critique d'homologation négative et prennent conscience de leur posture passée comme le déclare cet étudiant : "La première fois, la vidéo 2, je ne pensais pas que c'était de la danse, là oui.»(G2). La pratique a recentré leur regard sur le corps en mouvement, corps dont ils arrivent désormais à lire les intentions en termes de rapports aux fondamentaux (espace, temps, flux, poids, relation) : "C'est plus pareil. On regarde l'espace, les relations, le rythme, etc. » (G4). Ils disent avoir «vu plus de choses » (G3), avec un regard "plus axé sur la technique» (G5, G3, G2) et mieux comprendre la "construction de la chorégraphie » et "associer des termes techniques »(G2). L'évaluation esthétique (Château, 2010) émerge ici, les étudiants accédant aux propriétés de l'œuvre. Ces résultats vont dans le sens de Guisgand (2006) et plus généralement du lien pointé en neurosciences entre répertoire moteur et activation du système miroir de l'observateur (Rizzolatti \& al., 1996). Par ailleurs, deux étudiantes du même groupe (G4) nous déclarent même se mettre à la place des danseurs, tenter de comprendre les ressorts de l'interprétation, l'intention qui génère le mouvement. Ici, le lien entre expérience corporelle et sensible pendant la pratique et l'expérience esthétique en tant que spectatrice est clairement évoqué.

Ces résultats qui corroborent notre hypothèse ont pourtant des limites. Un étudiant du G2 nous dit avoir la même appréciation des vidéos mais être mieux équipé pour en parler. En effet, si le lien entre ces deux expériences peut se constater chez certains étudiants, nous en repérons aussi qui sont «plus respectueux » (G3, G2) mais qui ne s'abandonnent pas à l'expérience esthétique (au sens de Dewey, 1934/2005). Qu'est-ce qui fait alors obstacle?

\subsubsection{Les limites de cette évolution: de la difficulté s'abandonner l'expérience esthétique}


Nos données nous permettent d'apporter deux résultats quant aux limites de l'évolution des étudiants vers une expérience esthétique en mettant en lumière deux dimensions de la difficulté à s'abandonner à l'expérience esthétique : le rapport au divertissement et la difficulté d'accueillir certaines émotions. Comme nous l'avons évoqué, la question de l'homologation a presque disparu des échanges lors du deuxième visionnage, à l'exception du groupe 2 où trois étudiants reviennent, toujours en réaction à Café Müller, à ce que la danse « devrait être » selon eux :

E1 : Pour moi, c'est pas de la danse. Moi j'ai encore cette représentation de la danse, c'est plus bouger.

E2 : Là, c'est une répétition de mouvements dénuée de sens, c'est difficile.

E1 : Moi, par contre, je pense qu'il y a du sens mais que c'est pas de la danse. C'est plus une mise en scène, du théâtre.

Cher : Que serait la danse alors?

SILENCE

E1, après 6 secondes de réflexion : Il y a trop de signification, pour moi... là on pourrait sous-titrer... pour moi la danse... Là, c'est clairement dit, on ne peut pas interpréter autrement, on peut y voir quelque chose de différent mais on va tous se rapprocher du même sens alors que la danse chacun le voit comme il le sent. Pour moi, là, c'est trop...

E3 : Ouais, la danse, c'est plus des gens qui s'éclatent, qui ne cherchent pas à transmettre quelque chose.

E1 acquiesce.

Deux dimensions sont à prendre en considération quant à la résistance de ces étudiants face à la vidéo de Café Müller. Tout d'abord, la tension entre art et divertissement qui se clarifie lors de l'intervention de E3 à laquelle E1 acquiesce : la danse est envisagée comme divertissement, comme performance physique et non comme support de réflexion et d'expérience. Autour de cette tension, la notion de sens ${ }^{12}$ est omniprésente : là où il n'y a aucun sens pour E2 qui se retrouve confronté à une étrangeté impossible à appréhender, E1 et E3 condamnent la prégnance d'un sens qui nous oblige à la réflexion. Dans les deux cas, cependant, la résistance est la dynamique principale. Ensuite, il est intéressant d'approcher un deuxième aspect de cette résistance soulevé par E1 : dans Café Müller, le sens serait trop prégnant et ne permettrait que peu de différence d'interprétation. Cette assertion, imputable à la dimension "théâtrale » du courant danse-théâtre, est contredite par la profusion des lectures qu'ont pu en faire les étudiants (déshumanisation, inéluctabilité, rapport au cadre/à l'autorité/à la société, hôpital psychiatrique, Sisyphe, force des sentiments), profusion d'interprétations en lien avec la dimension «sur-assertive » de l'œuvre qui ne « dit» rien mais est « une concentration du sens présent de manière affaiblie et disséminée dans le contenu d'autres expériences » (Dewey, 1934 ed. 2005 , p. 157) ; cependant, il s'agit bien dans tous les cas d'une œuvre qui invite le spectateur à une expérience esthétique teintée d'émotions non jubilatoires, entrant en "interaction avec ce que le spectateur porte en lui » (ibid, p. 161). Faisant usage d'un langage surréaliste, la danse-théâtre de Pina Bausch "plonge le spectateur dans le vertige de ses propres angoisses » (Leroy, 2013, p.82). Et c'est bien cette dynamique de maturation et la prégnance de l'émotion qui l'accompagne qui peuvent faire obstacle pour le spectateur néophyte. Pour éclairer davantage la réception comme processus de maturation et sa dimension émotionnelle, observons d'autres moments d'échanges relatifs aux émotions.

Les échanges du G3 se focalisent sur la tension entre regard plus averti en termes d'analyse technique et émotions : une étudiante déclare que les outils acquis par la pratique lui permettent d'analyser la danse mais l'éloigne dans le même temps de l'émotion ${ }^{13}$, deux autres reçoivent différemment Café Müller et ressentent des émotions absentes ou différentes la première fois "même en analysant "; une quatrième utilise un "regard technique » sur les vidéos qui la touchent le moins alors que face à celle qui la touche le plus (Café Müller) elle déclare : «Ca me saisit, je me laisse aller à l'émotion». Ces propos nous donnent deux types d'indices sur la réception de la danse contemporaine par des novices : le fait que la pratique a bien un impact sur le regard porté en termes de capacité à lire la danse, mais aussi que la sensibilité face à des œuvres majeures est un complexe entre lecture et acceptation des émotions que l'œuvre véhicule : par exemple un étudiant du G5 qui

12 Il s'agit plus exactement de signification au sens de Zarifian (2000, p. 180) mais nous avons gardé ici le terme utilisé par les étudiants.

13 A noter : c'est la même étudiante qui parlait d'arnaque lors du premier visionnage. 
nous dit que malgré le regard plus technique il ressent toujours de l'angoisse face aux V2 et V3 et le vit comme négatif. Une autre (G5) est submergée par l'angoisse de mort à laquelle Café Müller la renvoie. Il est difficile pour les étudiants de considérer que l'art est là pour nous interroger, y compris sur des dimensions teintées d'émotions vécues comme non jubilatoires. Deux extraits pourraient être cités, tellement l'état des étudiants est palpable (Café Müller, Visionnage 2, G5) : " Ca me faisait peur, des souvenirs... enfin pas à moi, mais des souvenirs tristes "; "on dirait des mortes... elle si maigre... et l'autre tout à l'heure qu'on mettait dans les bras comme... ». Ces échanges nous rappellent que l'expérience esthétique telle que la décrit Dewey (1934 ed. 2005) est le contraire de la stase et que le phénomène de maturation n'est pas toujours sans violence, car «il y a peu d'expériences esthétiques intenses qui soient entièrement jubilatoires » (ibid, p. 90). Mais, comme l'écrit cet auteur, «La lutte et le conflit peuvent être appréciés en tant que tels en dépit de la souffrance qu'ils provoquent, quand ils sont vécus comme des moyens de faire avancer une expérience (...) qu'ils ne se contentent pas d'être là uniquement » (ibid, p. 89). Nous pensons que le problème se situe bien à ce niveau pour nombre d'étudiants, et la prégnance des échanges relatifs à Café Müller est d'ailleurs une donnée qui va dans ce sens : si certains ne veulent pas dépasser le divertissement, d'autres ont un vécu difficile de la réception de cette œuvre car ils subissent leurs émotions et ne trouvent pas le ressort pour les intégrer dans un processus d'expérience, atteints de plein fouet et suffoqués (Leroy, 2013, p.82). Ils restent sidérés par l'émotion brute, subie, qu'ils jugent négative, jugement qu'ils étendent conjointement à l'œuvre entière, voire à l'art en général.

\section{Discussion : des pistes pour la formation}

Les résultats nous permettent d'affiner notre compréhension de la réception chorégraphique chez les néophytes en distinguant mieux ce qui relève du voir et ce qui relève du percevoir et du recevoir. L'hypothèse qui a guidé cette recherche-intervention s'appuyait sur notre pratique et sur la notion d'expérience en particulier dans sa dimension relative à l'empathie et à la mémoire kinesthésique. Par l'expérience de la pratique corporelle et sensible, les étudiants ont majoritairement dépassé le stade de l'homologation qui était centrale lors du premier visionnage ; de plus, ils ont massivement établi un lien entre leur vécu corporel et sensible lors des ateliers et leur lecture du mouvement désormais attentive aux paramètres fondamentaux du mouvement. Ainsi, ce qui a clairement évolué, c'est leur capacité à voir la danse, à prendre "le mouvement dansé comme fil conducteur et comme point de focalisation du regard » (Guisgand, 2006, p.118) ce qui était notre hypothèse.

Pour autant, certains n'ont pas eu la sensation que cette appréhension plus fine contribuait à la réception de l'œuvre. Nos résultats ont permis de faire émerger deux obstacles à ce lien entre voir et perception - réception. Tout d'abord, l'approche de l'art chorégraphique (et peut-être de l'art en général) comme subordonné au langage, à l'interprétation et à la recherche de signification, ce qui rejoint les travaux de Ginot (2006) et de Montaignac (2014) : les étudiants, lors du deuxième visionnage, disent focaliser leur regard sur le corps en mouvement mais évoquent toujours la question de la signification, même en positif ( «j'ai mieux compris »). Dans le même temps, la question des émotions a été prégnante, dans leur lien à la perception mais aussi et surtout quant à l'acceptation de l'expérience à laquelle elles nous invitent. En effet, si certains étudiants ont amorcé un véritable travail relevant de l'expérience sur leur posture de spectateur en alliant perception et émotions pour atteindre une reconstruction de leur rapport au monde (Dewey, 1934 ed. 2005, p. 90), d'autres sont restés relativement froids face aux vidéos, comme lors du premier visionnage, malgré leur lecture plus fine du mouvement dansé. Nous pouvons ici reconnaître la distinction que décrit Dewey entre réception, perception et reconnaissance, cette dernière restant engluée dans des conventions et la recherche de gratification sensorielle, ne permettant pas de percevoir ni de recevoir, la perception impliquant la conscience, la réception impliquant l'abandon. Enfin, certains étudiants ont été submergés par des émotions sans parvenir à en dépasser la violence, en particulier pour l'extrait de Café Müller.

Du point de vue de la formation, et plus généralement d'un point de vue didactique, cette étude nous permet d'avancer quelques pistes. Tout d'abord, les résultats confirment que l'on peut considérer la réception chorégraphique comme une praxéologie en lien avec des savoirs sous-jacents de nature distinctes qui peuvent se construire dans un milieu d'étude et pour lesquels la notion d'expérience au sens de Dewey s'avère heuristique. Une approche de la danse comme expérience corporelle et esthétique (Huet et Gal-Petifaux, 2011 ; Dewey, 1934 ed. 2005) circonscrite mais aussi sensible et 
authentique ${ }^{14}$ que possible a un impact certain sur la réception dans sa dimension du « voir », savoir (comme «puissance d'agir») indispensable, premier, mais insuffisant : pour une expérience de réception, le processus de maturation reste tributaire de la volonté du sujet à s'y engager mais peut probablement être positivement influencé par une organisation des échanges en petits groupes où l'intervenant accompagne au besoin la mise en relation équilibrée entre agir et éprouver grâce à des jeux de langages permettant d'affiner la manière non seulement de parler mais aussi de sentir les œuvres (Michaud, 1999).

Ainsi, du point de vue épistémique et didactique, deux points fondamentaux à prendre en compte pour une formation à la réception des œuvres chorégraphiques : une pratique corporelle et sensible permettant de mieux lire la danse et d'amorcer une première sensibilité kinesthésique mais aussi un accompagnement basé sur un protocole tel que celui développé par Terrien (2006) pour l'écoute musicale : lors de visionnages successifs, exprimer librement son émotion puis chercher à la relier aux éléments chorégraphiques qui ont pu la susciter, privilégiant la construction du sens par la mise en relation des propriétés saillantes d'un passage chorégraphique avec les impressions ressenties. Cet accompagnement aurait toute sa pertinence et viserait à permettre de replacer les émotions dans un processus d'expérience où elles ne sont plus seulement subies.

\section{Bibliographie}

Arnaud-Bestieu, A. (2016). Formation et ressources praxéologiques de l'enseignant débutant en danse. Recherches en Éducation, HS 9, pp. 140-153.

Arnaud-Bestieu, A., Arnaud, G. (2013). La danse Flamenca, Paris : L'Harmattan.

Baribeau, C. (2009). Analyse des données des entretiens de groupe, Recherches qualitatives, 28, 133-148.

Bernard, M. (2001). De la création chorégraphique. Paris : Centre national de la danse.

Brousseau, G. (2003). Glossaire de quelques concepts de la théorie des situations didactiques en mathématiques [en ligne : pagesperso-orange.fr/daest/guy-brousseau/textes/Glossaire_Brousseau.pdf].

Château, D. (2010). L'expérience esthétique. Rennes : PUR.

Chevallard, Y. (1999). L'analyse des pratiques enseignantes en théorie anthropologique du didactique. Recherches en didactique des mathématiques, 19(2).

Dewey, J.(1934 ed.2005). L'art comme expérience. Paris : Folio.

Dewey, J. (1938 ed 2011). Expérience et éducation. Paris : Armand Colin.

Fratagnoli, F. (2010). Les danses savantes de l'Inde à l'épreuve de l'Occident. Thèse de Doctorat, Université Paris 8 , non publiée.

Germain-Thomas P. et Pagès D. (2014). Médiation et médiatisation en danse contemporaine : quand la profusion opacifie le sens d'un art sans texte. Quaderni, 83, 41-57.

Garcia, M-C., Cogérino, G., Fouilhoux, B. (2013). Regards sur le sensible, Staps, 102, 7-13.

Ginot, I. (2006). La critique en danse contemporaine, théories et pratiques, pertinences et délires, HDR, Paris 8 , non publié.

Guillemette F. et Lapointe, J-R. (2011). L'autoformation du stagiaire, in Guillemette F. et Lhostie, M, Favoriser la progression des stagiaires en enseignement, Québec, PUQ, 5-33.

Guisgand,P. (2006). Réception du spectacle chorégraphique : d'une description à l'analyse esthétique, Staps, 4.

Guisgand, P. (2014). Réception esthétique et pratique dévolutive, Recherches en Danse, 1.

Huet B. et Gal-Petitfaux N. (2011). L'expérience corporelle. Paris : Éditions EPS.

Laban (1947 ed. 2003). La danse moderne éducative. Arles : Actes Sud.

Laban (1950 ed.1994). La maîtrise du mouvement. Arles : Actes Sud.

14 Nous entendons par le terme « authentique » la proposition d'un travail au plus proche de la pratique sociale de référence. 
Leroy, C. (2013). Empathie kinesthésique, danse-contact-improvisation et danse-théâtre, Staps, 102, 75-88.

Massot-Leprince, M. (2016). Enseigner une discipline sans la maîtrise : exemple de la danse à l'école élémentaire. Thèse de Doctorat, Université de Nantes. En ligne:http://www.theses.fr/2014NANT3003.

Mauss, M. (1936). Les techniques du corps, Journal de psychologie, 3-4.

Mayen, G. (2005). Relation (auto)critiques, in Quant à la danse, Images en Manœuvres Éditions, 46-48.

Merleau-Ponty, M. (1945). Phénoménologie de la perception. Paris : Gallimard.

Michaud, Y. (1999). Critères esthétiques et jugement de goût. Nîmes : Editions Jacqueline Chambon.

Montaignac, K. (2014). Une anti-méthode ? Pour une analyse esthétique indisciplinée des œuvres chorégraphiques, Recherches en danse, 1.

Montaud, D. (2014). Le rapport aux ouvres dans l'enseignement de la danse au collège : analyse didactique de l'évolution de l'épistémologie pratique d'un professeur d'éducation physique et sportive, Thèse de Doctorat, Toulouse le Mirail, en ligne.

Reuter, Y. et al. (2007). Dictionnaire des concepts fondamentaux des didactiques. Bruxelles : De Boeck.

Rizzolatti, G., Fadiga, L., Gallese, V., Fogassi, L. (1996). Premotor cortex and the recognition of motor actions, Cognitive brain research, 3, 131-141.

Sensevy G., Mercier, A. (2007). Agir ensemble. L'action conjointe du professeur et des élèves dans la classe. Rennes : PUR.

Sensevy, G. (2008). Le travail du professeur pour l'action conjointe en didactique, Recherche et Formation, 57, 3950.

Terrien, P. (2006). L'écoute musicale au collège. Fondements anthropologiques et psychologiques. Paris : L'Harmattan.

Zakhartchouk, J-M.(2005). L'enseignant, un passeur culturel. Communication aux 3èmes Rencontres Cdi-Doc, Bordeaux, octobre 2005.

Zarifian, P. (2000). L’apprentissage par les événements : entre sens et signification. In Barbier, J-M. et Galatanu, O. Signification, sens, formation, Paris: PUF, 167-186.

\section{Annexe 1 : Situations de pratique}

\section{Séance 1 : Improvisations}

1. Mise en disponibilité : marcher dans la salle en occupant harmonieusement l'espace ; marcher à différentes vitesses, jusqu'à la course

2. Statues : alternance déplacement et arrêt subi dans une posture. Recherche immobilité et originalité des postures (flexibilité dos, tête, appuis inusuels)

3. Statues enroulées : un danseur en statue, un autre s'enroule autour.

4. La bulle : rendre visible sa kinesphère en la « touchant » devant, derrière, au- dessous et au-dessus avec les mains mais aussi les pieds, la tête, les fesses.

5. Les empreintes : faire des empreintes différentes avec différentes parties du corps en contact avec le sol.

6. La partie du corps ensorcelée : imaginer que sont ensorcelés la main, la tête, le bassin et qu'ils nous entraîne dans un déplacement dynamique, y compris au sol, en l'air...

7. Le musée : un tiers du groupe compose un espace de statues immobiles ; un autre tiers se déplace dans ce musée, en puisant dans des façons d'appréhender le déplacement expérimentées en amont (bulle/kinesphère, empreintes au sol ou déséquilibre entraîné par une partie du corps), et copie à l'envie les statues du musée en jouant sur les distances. Le troisième tiers : spectateurs. Suit un échange sur les ressentis.

8. Le fil du regard : par deux, évoluer dans la salle (bulle, empreintes, partie ensorcelée) sans rompre le fil extensible reliant les deux regards.

10. Sculpteur-sculpture : par 2, l'un sculptant le corps de l'autre sans utiliser les mains.

11 : Le musée sculpté : comme le musée mais les visiteurs peuvent modifier par contact les statues durant leur visite. 


\section{Séance 2: Vers la composition}

1. Mise en disponibilité : Alternance marche et cellule (s'étirer, fondre, se déposer, s'étaler, s'étirer, se recroqueviller, dérouler) à différentes vitesses.

2. Composer son mouvement : rendre visible sa kinesphère (départ et fin : position neutre).

3. Tableau ralenti-accéléré : après avoir explorer l'exécution de ce mouvement à l'extrême ralenti et à l'extrême accéléré, la moitié des étudiants se place dans l'espace de danse et chacun répète 5 fois ce mouvements, 3 fois en accéléré et au moins 1 fois au ralenti (la 5ème répétition étant libre) dans un ordre non imposé. La consigne est la recherche des extrêmes de vitesse. Les autres étudiants sont spectateurs. Suit un échange sur les ressentis.

4. Recherche de contrastes : avec ce même mouvement, en caressant l'espace/en giflant l'espace, comme un cosmonaute/comme sur un aimant, comme enfermé dans un placard à balais / en voulant toucher les murs et le plafond.

5. Recherches de positions en contre-poids et de portés en trio ou quatuor.

6. Composition de groupe à partir de textes littéraires.

Annexe 2 : Tableau de condensation des échanges, premier visionnage

\begin{tabular}{|c|c|c|c|c|}
\hline G1 & G2 & G3 & G4 & G5 \\
\hline $\begin{array}{l}\text { Opposition V1 (ra- } \\
\text { fraîchissant) et V2 et } \\
\text { V3 (angoissant) }\end{array}$ & $\begin{array}{l}\text { Est-ce que c'est de la } \\
\text { danse? N'importe } \\
\text { quoi }\end{array}$ & $\begin{array}{l}\text { Ne voit pas de sens, } \\
\text { ne peut pas apprécier }\end{array}$ & $\begin{array}{l}\text { V2 : redondant, lassi- } \\
\text { tude, incompréhen- } \\
\text { sion du sens }\end{array}$ & $\begin{array}{l}\text { Extraits éloignés de } \\
\text { l'image de la danse } \\
\text { (grâce, pureté, etc) }\end{array}$ \\
\hline V3 : Asile & $\begin{array}{l}\text { Art contemporain : li- } \\
\text { berté, expression, etc }\end{array}$ & $\begin{array}{l}\text { V2 : angoissant mais } \\
\text { plus intéressant que } \\
\text { V1, subjectivité }\end{array}$ & $\begin{array}{l}\text { V2 : drôle puis lassi- } \\
\text { tude }\end{array}$ & $\begin{array}{l}\text { Décalage, pas } \\
\text { d'émotion, pas beau, } \\
\text { bizarre }\end{array}$ \\
\hline $\begin{array}{l}\text { V3 : prison, V2: } \\
\text { crescendo de l'an- } \\
\text { goisse }\end{array}$ & $\begin{array}{l}\text { Différence avec le } \\
\text { ballet : plus organisé } \\
\text { mais moins d'émo- } \\
\text { tions }\end{array}$ & $\begin{array}{l}\text { V3 : agréable, salle } \\
\text { de classe }\end{array}$ & $\begin{array}{l}\text { V2 : déshumanisation } \\
\text { des interprètes, pas } \\
\text { maîtres de leurs } \\
\text { gestes, marionnettes }\end{array}$ & $\begin{array}{l}\text { V1 : classique mais } \\
\text { codes cassés }\end{array}$ \\
\hline $\begin{array}{l}\text { V2 et V3: mal à } \\
\text { l'aise }\end{array}$ & $\begin{array}{l}\text { Lien entre pratique } \\
\text { personnelle et récep- } \\
\text { tion DES danses }\end{array}$ & $\begin{array}{l}\mathrm{V} 2 \text { : corps qui est } \\
\text { musique }\end{array}$ & Marionnettes : V1 & $\begin{array}{l}\text { V2 et V3 : Impres- } \\
\text { sion de mouvements } \\
\text { mis bout à bout, ridi- } \\
\text { cule }\end{array}$ \\
\hline $\begin{array}{l}\text { V2 : plus du théâtre } \\
\text { que de la danse, en- } \\
\text { nuyeux }\end{array}$ & $\begin{array}{l}\text { Danse contemporaine } \\
\text { loin de nous, difficul- } \\
\text { té }\end{array}$ & V3 : psychiatrie. & $\begin{array}{l}\text { V2: inéluctabilité, } \\
\text { répétition, volonté }\end{array}$ & $\begin{array}{l}\text { V2 : opposition rai- } \\
\text { deur/rondeur : sen- } \\
\text { sualité, passion }\end{array}$ \\
\hline $\begin{array}{l}\text { V2 : troisième } \\
\text { homme, angoissant }\end{array}$ & $\begin{array}{l}\text { Liberté du créateur } \\
\text { mais aussi du specta- } \\
\text { teur, plus d'identifi- } \\
\text { cation que dans ballet }\end{array}$ & $\begin{array}{l}\text { Émotion avant sens, } \\
\text { Sens pas nécessaire }\end{array}$ & $\begin{array}{l}\text { Évocation d'autres } \\
\text { arts. V1 : théâtre, } \\
\text { V2 : peinture, V3 : } \\
\text { cinéma }\end{array}$ & V2 : force du silence \\
\hline $\begin{array}{l}\text { Impression que ce } \\
\text { n'est pas de la danse. } \\
\text { V2 : angoisse }\end{array}$ & $\begin{array}{l}\text { Subjectivité des émo- } \\
\text { tions, recherche de } \\
\text { l'intention du créa- }\end{array}$ & V3: sexualité & $\begin{array}{l}\text { V1 : technique, danse } \\
\text { V2 et V3 : pas de dé- }\end{array}$ & $\begin{array}{l}\text { Hermétique, il faut } \\
\text { être initié }\end{array}$ \\
\hline
\end{tabular}




\begin{tabular}{|c|c|c|c|c|}
\hline & teur & & placement & \\
\hline $\mathrm{V} 2$ : triste & $\begin{array}{l}\text { V1 : parodie, désa- } \\
\text { cralisation }\end{array}$ & $\begin{array}{l}\text { V3 : accélération, an- } \\
\text { goisse }\end{array}$ & $\begin{array}{l}\text { V1: danse narrative } \\
\text { V2 : désordonné }\end{array}$ & $\begin{array}{l}\text { V2: tristesse, pou- } \\
\text { pées de chiffon }\end{array}$ \\
\hline \multirow[t]{8}{*}{$\begin{array}{l}\text { V1 : ma représenta- } \\
\text { tion de ce qu'est la } \\
\text { danse }\end{array}$} & $\begin{array}{l}\mathrm{V} 2: \text { satyre drama- } \\
\text { tique anticonformiste }\end{array}$ & $\begin{array}{l}\text { Lien avec théâtre, } \\
\text { ressort physique } \\
\text { V2 : amour plus puis- } \\
\text { sant }\end{array}$ & $\begin{array}{l}\text { V1 : comique } \\
\text { V2 et V3 : pas drôle }\end{array}$ & $\begin{array}{l}\text { V2 : marionnettes, } \\
\text { rentrer dans un cadre }\end{array}$ \\
\hline & $\begin{array}{l}\text { V2: Tristesse, lour- } \\
\text { deur, oppression }\end{array}$ & V3 : hypnotique & $\begin{array}{l}\text { V3 : humanité } \\
\text { (mêmes choses mais } \\
\text { à différents moments } \\
\text { de la vie) }\end{array}$ & $\begin{array}{l}\text { V2 et V3 : mise en } \\
\text { avant de l'incons- } \\
\text { cient. V2 }: \text { autorité } \\
\text { V3 : agression et } \\
\text { rapport à la sexualité }\end{array}$ \\
\hline & $\begin{array}{l}\mathrm{V} 2 \text { et V3 :Déçue par } \\
\text { dénuement, arnaque }\end{array}$ & $\begin{array}{l}\text { Ennui, préfère danses } \\
\text { «plus époustou- } \\
\text { flantes » (hip-hop) }\end{array}$ & $\begin{array}{l}\mathrm{V} 2 \text { et } \mathrm{V} 3 \text { : interpréta- } \\
\text { tion personnelle }\end{array}$ & $\begin{array}{l}\text { V3 : féminité stéréo- } \\
\text { typée, sexualité }\end{array}$ \\
\hline & $\begin{array}{l}\mathrm{V} 2 \text { : théâtre, comique } \\
\text { de répétition, ridicule }\end{array}$ & $\begin{array}{l}\text { Pas danse comme on } \\
\text { s'y attendait }\end{array}$ & $\begin{array}{l}\text { V2 et V3 : artistes } \\
\text { engagées }\end{array}$ & V3 : folie \\
\hline & $\begin{array}{l}\text { Inaccessible quand } \\
\text { pas les codes }\end{array}$ & $\begin{array}{l}\text { V1 : comique, come- } \\
\text { dia dell'arte }\end{array}$ & $\begin{array}{l}\text { V2 : société, règles } \\
\text { extérieures imposées }\end{array}$ & $\begin{array}{l}\text { Ouverture d'esprit } \\
\text { nécessaire }\end{array}$ \\
\hline & $\begin{array}{l}\mathrm{V} 2 \text { :Pas les codes } \\
\text { mais ressent la souf- } \\
\text { france }\end{array}$ & $\mathrm{V} 2:$ tragique & $\begin{array}{l}\text { V3 : folie, aliénation, } \\
\text { camisole (chaise) }\end{array}$ & $\begin{array}{l}\text { Liberté d'interpréta- } \\
\text { tion }\end{array}$ \\
\hline & $\begin{array}{l}\text { V2 : métaphore so- } \\
\text { ciété }\end{array}$ & & $\begin{array}{l}\text { V1 : légèreté, V2 et } \\
\text { V3 : violence }\end{array}$ & \\
\hline & $\begin{array}{l}\text { V3 : hôpital psychia- } \\
\text { trique }\end{array}$ & & & \\
\hline & $\begin{array}{l}\text { V3 : critique société } \\
\text { (journées répétitives) }\end{array}$ & & & \\
\hline
\end{tabular}




\begin{tabular}{|c|c|c|c|c|}
\hline G1 & G2 & G3 & G4 & G5 \\
\hline $\begin{array}{l}\text { Changement de re- } \\
\text { gard sur la V2 (« bra- } \\
\text { quée » vers émo- } \\
\text { tions) }\end{array}$ & $\begin{array}{l}\text { Comprend mieux la } \\
\text { démarche. Mieux } \\
\text { compris, par ex V2. }\end{array}$ & $\begin{array}{l}\text { Des choses perçues } \\
\text { comme la première } \\
\text { fois : la V1. V2 : ré- } \\
\text { pétition comme la } \\
\text { 1ere fois mais effet } \\
\text { de souffrance. V3 : } \\
\text { unisson, rythme. } \\
\text { Maintenant mots, } \\
\text { première fois naïveté. }\end{array}$ & $\begin{array}{l}\text { «C'est plus pareil. } \\
\text { On regarde l'espace, } \\
\text { les relations, le } \\
\text { rythme, etc» }\end{array}$ & $\begin{array}{l}\text { Plus regarder, regard } \\
\text { plus sur la technique, } \\
\text { unisson, sentiments, } \\
\text { plus que moche, } \\
\text { beau, ridicule. }\end{array}$ \\
\hline $\begin{array}{l}\text { V2 : Effet de la répé- } \\
\text { tition: panique, } \\
\text { désordre, tristesse }\end{array}$ & $\begin{array}{l}\text { Association de } \\
\text { termes techniques et } \\
\text { compréhension de la } \\
\text { construction choré- } \\
\text { graphique }\end{array}$ & $\begin{array}{l}\text { Avec pratique, vu } \\
\text { plus de choses. Sur- } \\
\text { tout V3. }\end{array}$ & $\begin{array}{l}\text { 1ere fois plus dans } \\
\text { émotion, là plus tech- } \\
\text { nique. V3 : unissons } \\
\text { et décalages « Quel } \\
\text { travail! » }\end{array}$ & $\begin{array}{l}\text { V2 : 1ere fois ridi- } \\
\text { cule, là désespoir, } \\
\text { contraste, vitesses, } \\
\text { plus axée sur la tech- } \\
\text { nique }\end{array}$ \\
\hline $\mathrm{V} 2:$ tristesse & $\begin{array}{l}\text { Apprécié les } 2 \text { fois } \\
\text { mais mesure davan- } \\
\text { tage la prouesse phy- } \\
\text { sique. }\end{array}$ & 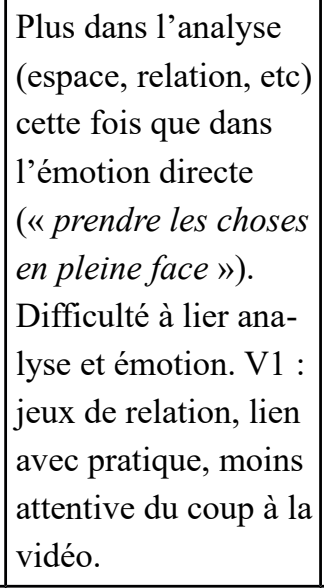 & $\begin{array}{l}\mathrm{V} 1: \text { lere fois co- } \\
\text { mique, là technique, } \\
\text { composition }\end{array}$ & $\begin{array}{l}\text { V2 : hôpital psychia- } \\
\text { trique avec des ma- } \\
\text { lades et des soi- } \\
\text { gnants }\end{array}$ \\
\hline $\begin{array}{l}\text { A la demande de C : } \\
\text { V1 ? } \\
\text { Burlesque, porters, } \\
\text { rythme, dynamique }\end{array}$ & $\begin{array}{l}\text { Même appréciation } \\
\text { mais mieux équipé } \\
\text { pour en parler. }\end{array}$ & $\begin{array}{l}\text { Premières fois : im- } \\
\text { pressions, là regard } \\
\text { plus technique }\end{array}$ & $\begin{array}{l}\text { V1 : 1ere fois lou- } \\
\text { foque, là les pieds, la } \\
\text { difficulté, les rela- } \\
\text { tions. V2 }: \text { lere fois } \\
\text { amour, là violence. } \\
\text { V3 : le rythme }\end{array}$ & $\begin{array}{l}\text { V2 : très prenant, } \\
\text { mal à l'aise. « Ca } \\
\text { me faisait peur, des } \\
\text { souvenirs... enfin } \\
\text { pas à moi, mais des } \\
\text { souvenirs tristes» }\end{array}$ \\
\hline $\begin{array}{l}\text { Première fois avait } \\
\text { vu du lent et rapide. } \\
\text { Maintenant énergie } \\
\text { du mouvement (ca- } \\
\text { resser/gifler) }\end{array}$ & $\begin{array}{l}\text { Plus de termes tech- } \\
\text { niques et mieux équi- } \\
\text { pés pour regarder }\end{array}$ & $\begin{array}{l}\text { Sur video qui tou- } \\
\text { chait moins regard } \\
\text { technique mais sur } \\
\text { V2 (touche le plus) } \\
\text { émotions. «Ca me } \\
\text { saisit, je me laisse al- } \\
\text { ler à l'émotion» }\end{array}$ & $\begin{array}{l}\text { Se met à la place des } \\
\text { danseurs, comprendre } \\
\text { en moi comment on } \\
\text { interprète pas seule- } \\
\text { ment spectateur. }\end{array}$ & $\begin{array}{l}\text { V3 : aliénation, unis- } \\
\text { son, unisson brisé, } \\
\text { retour à l'unisson. } \\
\text { Observation froide, } \\
\text { neutre }\end{array}$ \\
\hline
\end{tabular}




\begin{tabular}{|c|c|c|c|c|}
\hline $\begin{array}{l}\text { V3 : première fois } \\
\text { sensualité ; mainte- } \\
\text { nant non, répétition, } \\
\text { changements de vi- } \\
\text { tesses et d'énergies }\end{array}$ & $\begin{array}{l}\mathrm{V} 2 \text { : première fois ne } \\
\text { pensait pas que } \\
\text { c'était de la danse, là } \\
\text { oui. }\end{array}$ & $\begin{array}{l}\mathrm{V} 2: \text { première fois } \\
\text { pas touchée, là oui, } \\
\text { même en analysant }\end{array}$ & $\begin{array}{l}\text { Question de l'inten- } \\
\text { tion qui génère le } \\
\text { mouvement, aller } \\
\text { chercher en soi pour } \\
\text { projeter le mouve- } \\
\text { ment. V3 : rappel de } \\
\text { gifler/caresser l'es- } \\
\text { pace }\end{array}$ & $\begin{array}{l}\text { V2 : étouffement, } \\
\text { étrangeté }\end{array}$ \\
\hline $\begin{array}{l}\mathrm{V} 3: \text { « Maintenant on } \\
\text { a des mots », pour } \\
\mathrm{V} 3: \text { première fois } \\
\text { rien vu, maintenant } \\
\text { saccades, contrastes, } \\
\text { etc } \\
\text { C : Cela influence } \\
\text { ton ressenti? } \\
\text { Pas V3 mais V2 oui }\end{array}$ & $\begin{array}{l}\text { V2 : pas de la danse, } \\
\text { «la danse, c'est plus } \\
\text { bouger » } \\
\text { V2 : là difficile, répé- } \\
\text { tition de mouvements } \\
\text { dénués de sens } \\
\text { V2 : sens mais } \\
\text { théâtre. Trop de si- } \\
\text { gnification } \\
\text { V2: danse « c'est } \\
\text { des gens qui } \\
\text { s'éclatent sur de la } \\
\text { musique, qui } \\
\text { cherchent pas à } \\
\text { transmettre quelque } \\
\text { chose » (l'autre étu- } \\
\text { diante acquiesce) }\end{array}$ & $\begin{array}{l}\text { Plus analyse que la } \\
\text { première fois. V2 : } \\
\text { première fois beau- } \\
\text { coup dérangé, là re- } \\
\text { gard différent }\end{array}$ & $\begin{array}{l}\text { Conscience du tra- } \\
\text { vail, beaucoup plus } \\
\text { que la } 1 \text { ere fois }\end{array}$ & V2 : tristesse \\
\hline & $\begin{array}{l}\text { Danse comme les } \\
\text { autres arts : peut être } \\
\text { plus sur la forme, le } \\
\text { sens ou l'émotion di- } \\
\text { recte. V2 : fond. Sy- } \\
\text { siphe, absurdité mais } \\
\text { peut aussi symboliser } \\
\text { force du sentiment }\end{array}$ & $\begin{array}{l}\text { Plus à-même de com- } \\
\text { prendre le travail, } \\
\text { plus respectueux }\end{array}$ & & $\begin{array}{l}\text { Regard plus tech- } \\
\text { nique mais ne } \\
\text { change pas regard, } \\
\text { émotion. V2 et V3 : } \\
\text { angoisse, mal à } \\
\text { l'aise, flippée... re- } \\
\text { connais plus la tech- } \\
\text { nique mais angoisse } \\
\text { toujours }\end{array}$ \\
\hline
\end{tabular}




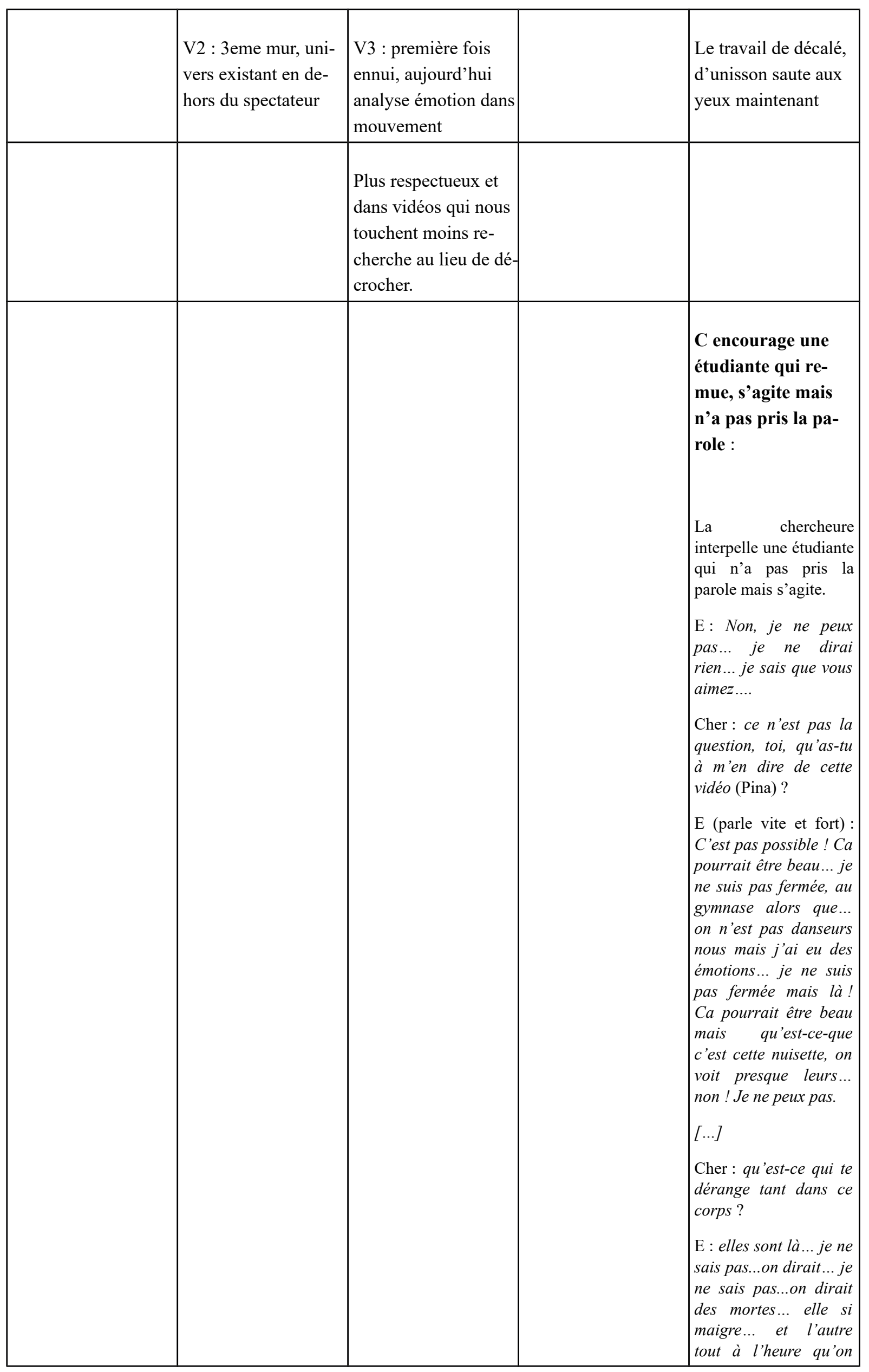




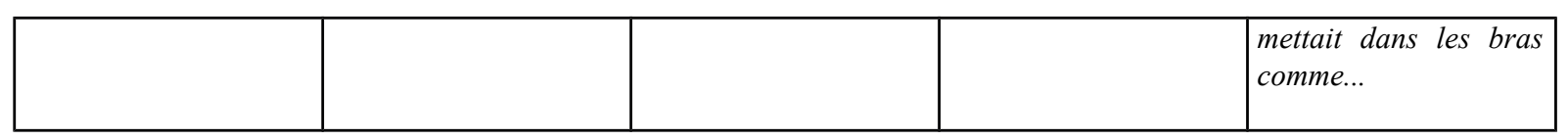

